\title{
Acciones para la formación inicial de la competencia profesional específica registrar hechos económicos.
}

\section{Work for the initial formation of the specific professional competition to} register economic facts.

\author{
Carlos Albero Carballosa. ${ }^{1}$, Elizabet Prado Chaviano. ${ }^{2}$, Cecilia Celestrín \\ Penabades. ${ }^{3}$, Bisleivys Jiménez. ${ }^{4}$ \& Luis Efraín Velastegui López. ${ }^{5}$
}

DOI: https://doi.org/10.33262/visionariodigital.v5i2.1720

\begin{abstract}
.
The initial training of the specific professional competences to register that economics is currently considered as a priority tasks to comet essential to guaranty the professional performances of graduated. The accounting an finances carrier in it consideration as a competences is conceded as a complex continues process and systemic interacted knowledge, skills, values, motivation and performances related to the analyses, processing of economic events for their subsequent registration in the mandatory accounting books in accordance with the accounting regulation solve problems in the territory and entity for with e system of action is propose implement by the economic departments of the group with the economics financial management, specialist who contribute to the professional improvement.
\end{abstract}

Keywords: Professional competences, register that economics.

\footnotetext{
${ }^{1}$ Universidad de la Isla de la Juventud, Isla de la Juventud, Cuba, email: carlos.carballosa@gmail.com, Orcid. http//orcid.org/0000-0002-9183-7505

${ }^{2}$ Universidad de Matanzas, Matanzas, Cuba. email: elizabetpradochaviano7979@gmail.com, Orcid. http//orcid.org/0000-0002-8101-0440

${ }^{3}$ Universidad de Matanzas, Matanzas, Cuba. Email: cecilia.celestrin@ umcc.cu Orcid. http//orcid.org/00000002-8719-069x

4 Universidad de Matanzas, Matanzas, Cuba. Email: bisleivys.jimenez@umcc.cu Orcid. http//orcid.org/0000--0003-4812-4558

${ }^{5}$ Ciencia Digital Editorial, Ecuador, luisefrainvelastegui@cienciadigital.org
} 


\section{Resumen.}

La formación inicial de la competencia profesional específica registrar hechos económicos se considera en la actualidad como una tarea de orden prioritario a acometer, imprescindible para garantizar el desempeño profesional de los egresados en la carrera Contabilidad y Finanzas. En su consideración como competencia se concibe, como un proceso continuo, complejo y sistémico que integra conocimientos, habilidades, valores éticos del contador, motivaciones y desempeños relacionados con el análisis y procesamiento de los hechos económicos para su posterior registro en los libros obligatorios de la contabilidad acorde con la normativa contable evidenciados en el entorno laboral. Esta investigación, sustenta dicha formación inicial, desde las tareas del perfil profesional para resolver problemas en las entidades del territorio, para lo cual se propone un sistema de acciones implementadas en los departamentos económicos de conjunto con los especialistas de Gestión Económico Financiera que contribuyeron en el mejoramiento profesional y humano de los estudiantes.

Palabras claves: Competencia profesional, registrar hechos económicos.

\section{Introducción.}

La formación de profesionales competentes en la Educación Superior Contemporánea es uno de los aspectos que en la actualidad merece ser estudiado, para lograr transformar a los educandos en verdaderos transformadores de su entorno y comprometidos con el desarrollo social, de acuerdo a los constantes cambios que se originan en los escenarios ocupacionales y educativos.

Las competencias profesionales en las Ciencias Contables y Financieras, ha sido en la actualidad un tema en estudio donde se reconocen competencias generales relacionadas con el trabajo en equipo, la negociación y gestión de proyectos, el análisis, recopilación de la información para el procesamiento de los datos, transmisión del conocimiento, innovación y aprendizaje, compromiso, iniciativa e identificación con la organización (Tobar, Arias y Ríos, 2018) y competencias específicas relacionadas con el manejo y archivo de los documentos mercantiles, registrar hechos económicos, el análisis de los estados financieros, la utilización de los software (Hernández y Fajardo, 2011; Muñoz, 2013; Hernández, 2014; Lemas, 2015; Linares y Suárez, 2017 y Rocancio, Mira y Muñoz, 2017).

En estas investigaciones anteriores sobre la competencia profesional específica registrar hechos económicos no se fundamenta la misma desde el punto de vista teórico, ni se estructuran las posibles vías de formación, solo se señalan algunas tareas a desarrollar y pruebas vinculadas a la preparación de los asientos de diarios en los libros obligatorios y auxiliares, según plan contable, las normas legales vigentes, que incluye, la verificación y conciliación de los hechos económicos.

Este estudio, sobre la formación inicial de la competencia profesional específica registrar hechos económicos se vincula a las exigencias del Plan de Estudio E, en relación a los modos de actuación, campos de acción y objetivos de la carrera, a través del registro 
contable del uso de los recursos materiales y financieros de las entidades, en correspondencia con las Normas Cubanas de Contabilidad y las Normas Internacionales de la Información Financiera así como las técnicas que rigen el proceso contable (MES, 2017) y contribuye a la presentación de la información económica financiera de manera veraz, porque un mal registro de los hechos económicos por descuido, desconocimiento, evasión de información o irregularidades no declaradas, puede conducir a la deformación de los resultados de los estados financieros, la toma de decisiones oportunas, los resultados no confiables en las auditorias y un insuficiente desempeño demostrado en el puesto de trabajo.

La formación inicial de la competencia profesional específica registrar hechos económicos integra sistemas de conocimientos relacionados con la normativa contable que desempeña un rol fundamental en la actividad contable (Di Napoli, 2011), a su vez se tratan las entidades como unidades económicas capaces de satisfacer las necesidades colectivas mediante la producción de bienes y servicios. De igual modo, se considera la documentación mercantil, que se refiere a toda la constancia escrita de una transacción comercial para certificar las anotaciones en los libros de contabilidad y probar un hecho desde el punto de vista legal, las cuentas y subcuentas como instrumentos de representación y medida de un elemento del patrimonio o de los resultados y los hechos económicos como un acto o transacción que, afecta al patrimonio de la entidad o afecta a las cuentas de resultados, lo cual se sustenta en el método de la Partida Doble, con la aplicación de su fundamento matemático, que divide la cuenta en dos secciones denominadas debe y haber para posteriormente, contabilizar los hechos económicos en el libro diario, partir de asientos contables.

El conocimiento de los procedimientos contables, debe tenerse en cuenta, porque describe a través de una secuencia de pasos el objetivo del procedimiento y alcance, los responsables de la ejecución del mismo, el archivo de los documentos mercantiles, las definiciones en relación a las cuentas o elementos a tener en cuenta de control interno, los hechos económicos, los modelos, resoluciones, las cuentas y subcuentas y los asientos tipos más utilizados en las entidades (Prado, 2017 y Prado, 2018a).

Asimismo, deben contemplarse los valores éticos del contador evidenciados en el registro de los hechos económicos, tales como: la honestidad, profesionalidad y fidelidad que se materializan en el correcto registro de los bienes y recursos que poseen las entidades, el cumplimiento de las normas internacionales y cubanas y la colaboración mediante el trabajo en equipo en el proceso de enseñanza y con los especialistas en el área contable en el entorno laboral.

Estos aspectos se materializan en el entorno laboral a través de tareas del perfil profesional en las entidades donde la motivación, ocupa un papel importante, sí como las relaciones interpersonales y el desempeño alcanzado en la identificación de los elementos necesarios del registro, las regularidades de las cuentas de dicho registro para diversas entidades, el comportamiento y otros componentes del modo de actuación profesional. 


\section{Metodologia.}

En la investigación se realiza un diagnóstico con el fin de identificar las limitaciones con respecto a las acciones realizadas en función de la formación inicial de la competencia profesional específica registrar hechos económicos, en la cual se aplica una observación a los estudiantes de la carrera Contabilidad y Finanzas para medir el conocimiento del registro de los hechos económicos en ambiente laboral, donde se conciben las categorías siguientes: MA muy adecuado, BA bastante adecuado, A adecuado, PA, I inadecuado. A continuación se presenta la tabla 1 con los aspectos observados y los resultados de las categorías.

Tabla 1. Aspectos observados y los resultados de las categorías para la medición del conocimiento del registro de los hechos económicos en ambiente laboral.

\begin{tabular}{|c|c|c|c|c|c|}
\hline Aspectos & M A & B A & A & P A & I \\
\hline $\begin{array}{l}\text { Registra los hechos económicos a partir de semejanzas y } \\
\text { diferencias para cualquier tipo de actividad en ambiente } \\
\text { laboral. }\end{array}$ & & & & $x$ & \\
\hline $\begin{array}{l}\text { Dominio de situaciones contables en entidades del territorio } \\
\text { en ambiente laboral. }\end{array}$ & & & & & $x$ \\
\hline $\begin{array}{l}\text { Dominio de software contable donde se registran los hechos } \\
\text { económicos en ambiente laboral. }\end{array}$ & & & & & $x$ \\
\hline $\begin{array}{l}\text { Dominio de las tareas establecidas sobre el registro de los } \\
\text { hechos económicos para su ejecución en ambiente laboral. }\end{array}$ & & & & $x$ & \\
\hline $\begin{array}{l}\text { Establece relaciones interpersonales adecuadas en el área } \\
\text { laboral, valores éticos del contador y demuestra motivación. }\end{array}$ & & & $x$ & & \\
\hline $\begin{array}{l}\text { Demuestra motivación para registrar hechos económicos y } \\
\text { pide las ayudas necesarias para mejorar la actividad. }\end{array}$ & & & & $x$ & \\
\hline $\begin{array}{l}\text { Demuestra poseer los valores éticos del contador en el } \\
\text { entorno laboral. }\end{array}$ & & & $x$ & & \\
\hline
\end{tabular}

Fuente: elaboración propia

Las observaciones se realizaron en las entidades más estables en su relación con la universidad, las que tienen convenios firmados desde hace algunos años y donde se reciben mayor cantidad de estudiantes en las tareas del perfil profesional en las entidades, en la cual, no se observó de manera sistemática e intencionada el registro de los hechos económicos por parte de los estudiantes, aunque debían realizar el registro de la inversión Financiera, así como mencionar los métodos de registro y acumulación de los costos. Esto se debe, a que en las entidades los software contables están protegidos y solo pueden tener acceso a sus módulos para el registro de los hechos económicos los especialistas del departamento económico, y en otros contextos de actuación, en este caso, en las tareas del perfil profesional para resolver situaciones que se presentan en las entidades, no registran los hechos económicos aunque realizan controles a las cuentas de Inventarios y Activos Fijos. En ambos casos, se limita el aprendizaje de los estudiantes en estas entidades lo cual incide en el interés, la motivación y el reconocimiento de las limitaciones y progresos en el aprendizaje, aunque de forma general, se establecen buenas relaciones interpersonales con el colectivo de trabajo y valores éticos del contador como responsabilidad, honestidad y profesionalidad y motivación en las tareas acometidas. Se constató que el 17,91\% (12) estudiantes, observaron cómo el especialista de Gestión 
Económica Financiera, realizó el registro, lo que demuestra un acercamiento a la práctica contable.

También se realiza una encuesta a los egresados de la carrera Contabilidad y Finanzas que laboran en entidades del territorio con el propósito de recoger información sobre la manera en que se registrar hechos económicos en las entidades y como tributaron las asignaturas de la carrera en su desempeño laboral.

Se les aplica la encuesta a 25 egresados de la carrera Contabilidad y Finanzas, ubicados en los municipios de Matanzas y Cárdenas, 15 se encuentran en el primer año de adiestramiento y 10 en el segundo.

En los resultados de la encuesta, el 100\% (25) egresados expresan que registran los hechos económicos siguientes: compras de Inventarios y Activos Fijos, acumulación, extracción y pago del salario, enmiendas comprobantes y fijación del Inventario. Esto demuestra que en la etapa de adiestramiento en la mayoría de las entidades le asignan a los egresados tareas relacionadas con las adquisiciones, bajas y transferencias de los equipos e Inventarios, así como los comprobantes para la acumulación extracción y pago a los trabajadores de los salarios; estas tareas se realizan constantemente y al final del mes, se elaboran el informe de fijación de Inventarios y el control de los Activos Fijos Tangibles por área, obviando otras tareas como el registro de los hechos económicos relacionados con los Activos, Pasivos, Capital, Ingresos y Gastos.

Todos los egresados expresan que los conocimientos adquiridos en las asignaturas de la carrera son útiles, porque sirven como soporte teórico para registrar hechos económicos. Aunque reconocen que se estudia el registro manual de los hechos económicos en entidades comercializadoras e industriales, consideran que no se sienten lo suficientemente preparados para registrar hechos económicos en cualquier tipo de entidad y tampoco tienen el adiestramiento necesario para operar con los software contables.

El 20\% (5) egresados utilizan como pasos para el registro de los hechos económicos, el análisis de los importes, la identificación de las cuentas, subcuentas, el análisis del hecho económico y el registro en los subsistemas de Contabilidad, mientras que el resto, no utiliza pasos o sea, el 80\% (20) estudiantes solo registran los hechos con un aprendizaje memorístico de los códigos de las cuentas y subcuentas.

Los resultados de la observación y las encuestas demuestran la pertinencia de la investigación por lo que se propone un sistema de acciones para la formación inicial de la competencia profesional específica registrar hechos económicos desde los modos de actuación profesional en entidades del territorio.

A continuación se presentan las acciones.

Acción 1. Entrenamiento en los puestos de trabajo con el propósito de adquirir conocimientos y habilidades relacionadas con los documentos mercantiles, el archivo y 
procesamiento de la documentación primaria, la observación de reportes contables y la dinámica en los subsistemas de Activos Fijos Tangibles, Inventarios, Ingresos, Cobros y Pagos y efectivo.

Acción 2. Control de los Inventarios en el almacén a través de la constatación de las unidades físicas en correspondencia con las cantidades de los submayores así como el procesamiento contable y ajustes de mermas, deterioros, faltantes, sobrantes, la fijación y rotación de los inventarios atendiendo a su clasificación de rápida rotación, de lento movimiento y ociosos.

Acción 3. Control de los Activos Fijos Tangibles realizado al final del mes por áreas de responsabilidad y registro de la depreciación acumulada así como los comprobantes relacionados con las bajas, traslados y altas, con el propósito de mantener actualizado los importes de estos activos así como su ubicación y estado.

Acción 4. Observación y verificación del cumplimiento de la normativa y los procedimientos contables en función del objeto de la organización, las particularidades del uso y contenido de las cuentas y el sector a que pertenece, con el propósito de aprender a fiscalizar los registros, documentos e información contable.

Acción 5. Registro de los hechos económicos mediante comprobantes de operaciones o asientos contables con el fin de que adquieran conocimientos, habilidades y desempeños en operar sistemas contables automatizados, clasificar los hechos económicos, insertar las cuentas, importes, explicaciones, fechas, subcuentas, partidas y análisis de los hechos económicos.

Acción 6. Participación en el cierre y apertura de un período contable con el propósito de observar el cierre de las cuentas nominales y el cierre transitorio y reapertura de las cuentas reales. Acción 7. Participar en la planificación y ejecución del presupuesto de la empresa y comentar las desviaciones de las cuentas.

Acción 8. Modelación del ciclo contable con las evidencias de los registros, estados financieros, documentos y participación en los consejos económicos

\section{Resultados.}

Las acciones se desarrollaron en el curso 2019-2020 con 65 estudiantes de la carrera Contabilidad y Finanzas de segundo año del curso diurno como parte de la tarea de impacto desempeñada en entidades del territorio y para la valoración de los resultados se realiza una tormenta de ideas y un test de comprobación con los estudiantes en el anexo 1 y especialistas de Gestión Económica Financiera de los departamentos económicos con el fin de debatir sobre sus percepciones y avances en términos de conocimientos, habilidades, desempeños y motivaciones en función de las acciones propuestas para contribuir a eliminar las dificultades detectadas en el diagnóstico. 
En la tormenta de ideas el 100\% de los participantes reconocen que las acciones trazadas contribuyen en la formación inicial de la competencia profesional específica registrar hechos económicos en la carrera Contabilidad y Finanzas y disminuyen las dificultades detectadas

El test de comprobación aplicado arroja que de los 65 estudiantes el 15.38\% (10) estudiantes, dominan los procedimientos contables y se apoyan en ellos al registrar hechos económicos de forma manual y/o software, así como los libros obligatorios de la contabilidad y la tipificación de los hechos económicos.

El 60\% (39) estudiantes, conocen las cuentas que intervienen en el registro de los hechos económicos en entidades industriales, comerciales y de servicios, así como la ubicación de los productos en el almacén y respectivamente el llenado de la documentación y saben tipificar los hechos económicos.

El 24.61\% (16) estudiantes, conocen cuál es el destino de los documentos mercantiles, y su tratamiento en términos de fiscalización; así como los cambios a realizar en cuanto a la nomenclatura de las cuentas para diferentes entidades, cómo comprobar la exactitud, prudencia y confiabilidad al registrar hechos económicos.

\section{Conclusiones}

- Se presentan como aspectos teóricos que caracterizan la formación inicial de la competencia profesional específica registrar hechos económicos los documentos mercantiles, la normativa y los procedimientos contables, las cuentas, los hechos económicos, el libro de diario general, la motivación, los modelos, resoluciones, las cuentas y subcuentas y los asientos tipos más utilizados en las entidades asó como los valores éticos del contador y el desempeño demostrado.

- El diagnóstico reveló que existen limitaciones en la formación inicial de la competencia profesional específica registrar hechos económicos con énfasis en operar sistemas contables automatizados por los accesos restringidos a los software por seguridad de las empresas.

- Las acciones implementadas contribuyeron en la formación inicial de la competencia profesional específica registrar hechos económicos constatados en el test de comprobación aplicado a los estudiantes.

\section{Referencias bibliográficas.}

Di Napoli, F. (2011). El impacto de los cambios normativos internacionales en la formación de los contadores públicos (Tesis de maestría, Universidad de San Andrés, Argentina).

Hernández, G. C. (2014). Las competencias profesionales y la gestión de su mejora en procesos contables. Acercamiento teórico (CD-ROM). Matanzas. Universidad de Matanzas Camilo Cienfuegos. 
Hernández, G. C., y Fajardo, I. (2011). Sistema de actividades para contribuir a la formación de la competencia profesional para el análisis en estudiantes de la carrera Contabilidad y Finanzas en la FUM de Colón (CD -ROM). Matanzas. Universidad de Matanzas Camilo Cienfuegos.

Lemas, B. (2015). La planificación curricular por competencias y su incidencia en el proceso de enseñanza aprendizaje en la asignatura de proceso contable del bachillerato técnico en Contabilidad en la Unidad Educativa Intercultural Bilingüe Abya - Yala (Tesis de maestría, Universidad Técnica de Ambato, Ecuador).

Linares, M., y Suárez, Y. M. (2017). Competencias del contador público. Una mirada desde la Educación Superior y los requerimientos de las PYME comerciales en Bogotá. Revista Teuken Bidikay 8(10), p. 149-175. Recuperado de: http//:www. Researchgate.net/publication/322930197_COMPETENCIAS_DEL_COTADOR -PUBLICO-una-

mirada_desde_la_Educación_Superior_y_los_requerimientos_de_ las_pymes_comerciales_en_Bogotá.

MES. (2017). Plan de Estudio E. La Habana (Cuba).

Muñoz, S. (2013). Evaluación de las competencias del profesional contable egresado de la Universidad de Antioquia para desempeñarse en el sector financiero de Medellín. Revista Udea, 7 (1), sp. Recuperado de. http//revistas.uudea.edu.co.

Prado, E. (2017). Hiperambiente de aprendizaje para a formaçäo da competencia registar factos econòmicos. En J.N, Silvestre (Presidencia), IV Jornada Científico Pedagógica da Faculdade de Economía. Jornada dirigida pela Universidade Katyavala Bwila. Benguela.

Prado, E. (2018a). Procedimento para a formaçäo da competencia registar factos económicos asociados ao Inventario. En M.J, da Costa (Presidencia), IV Jornada Científica Pedagógica. Jornada dirigida pelo Instituto Superior da Ciencias da Educaçäo. Huambo.

Rocancio, A. D., Mira, G. E., y Muñoz, N. M. (2017). Las competencias en la formación del profesional contable. Una revisión de las posturas institucionales y educativas en Colombia. Revista De La Facultad De Ciencias Económicas, 12(2), p. 83-103. doi: https://10.18359/rfce.3070.

Tobar, G., Arias, M., y Ríos, G. (2018). Competencias del contador-auditor en el perfil de egreso. Revista de Actualidad Contable Faces, 21(37), p. 2-56. Recuperado de: http//:www.redalyc.org/artículo.oa?id=25755483005. 


\section{PARA CITAR EL ARTÍCULO INDEXADO.}

Albero Carballosa, C., Prado Chaviano, E., Celestrín Penabades, C., Jiménez Valero, B., \& Velastegui López, L. E. (2021). Acciones para la formación inicial de la competencia profesional específica registrar hechos económicos. Visionario Digital, 5(2), 216-224. https://doi.org/10.33262/visionariodigital.v5i2.1720

\section{Ciencia \\ LDigital}

El artículo que se publica es de exclusiva responsabilidad de los autores y no necesariamente reflejan el pensamiento de la Revista Visionario Digital.

El artículo queda en propiedad de la revista y, por tanto, su publicación parcial y/o total en otro medio tiene que ser autorizado por el director de la Revista Visionario Digital.
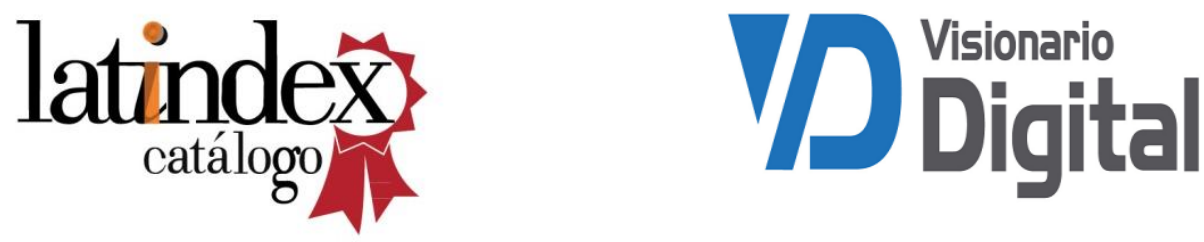